\title{
Breaking Diffeomorphism Invariance and Tests for the Emergence of Gravity
}

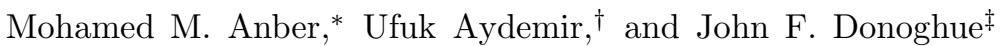 \\ Department of Physics, University of Massachusetts \\ Amherst, MA 01003, USA
}

\begin{abstract}
If general relativity is an emergent phenomenon, there may be small violations of diffeomorphism invariance. We propose a phenomenology of perturbatively small violations of general relativity by the inclusion of terms which break general covariance. These can be tested by matching to the Parameterized Post Newtonian (PPN) formalism. The most sensitive tests involve pulsar timing and provide an extremely strong bound, with a dimensionless constraint of order $10^{-20}$ relative to gravitational strength.
\end{abstract}

\section{INTRODUCTION}

Most approaches to new theories invoke higher gauge symmetries beyond those seen at low energies, with the known symmetries being respected at all energies. However, it is also possible that the gauge theories in nature are emergent, low energy manifestations from a fundamental theory with quite different degrees of freedom and perhaps without an intrinsic gauge invariance. Emergent phenomena are common in nature. While there may be obstacles to emergent mechanisms for fundamental theories, the possibility certainly deserves study.

Gravitational physics is a good candidate for an emergent theory because of the poor high energy behavior of general relativity. While the low energy theory forms a good quantum effective field theory [1] , at high energies the perturbative theory falls apart. This may signal the need for new degrees of freedom and new interactions beyond the Planck scale. In some theories, those with a background independence or explicit general covariance, the emergent gravitational theory will fully respect diffeomorphism invariance 2]. However, other candidate may not have this feature. There are several attempts to produce emergent gauge theories and gravity [3], with many using ideas based on condensed matter emergent analogies, as well as recent work on Horava-Lifshitz theories [4]. Indeed, the Witten-Weinberg theorem [5] implies that if emergence is to explain all the gauge theories, spacetime and Lorentz invariance may need to be emergent.

Emergent theories that start from a framework without diffeomorphism invariance will leave behind an imprint of the lack of this invariance. By definition, these theories reduce to general relativity at low energies, plus small corrections. At the very least, loop diagrams probe the very highest energies and will be sensitive to the lack of the invariance at the fundamental scale.

Gravity is also a good place for emergent phenomenology. The gravitational interactions are weak, suppressed

\footnotetext{
*Email: manber@physics.umass.edu

${ }^{\dagger}$ Email: uaydemir@physics.umass.edu

‡Email: donoghue@physics.umass.edu
}

by powers of the Planck scale. Therefore, it is plausible that the residual effects of non-invariance will be relatively more visible in gravitational interactions.

Motivated by these considerations, we will initiate a phenomenological study of potential small breaking of diffeomorphism invariance. While we do not know the underlying theory and therefore do not know the magnitude and form of the symmetry breaking, we study an effective Lagrangian with terms that break diffeomorphism invariance, and proceed to match on to the Parameterized Post-Newtonian (PPN) framework in order to provide a remarkably stringent bound.

The plan of this work is as follows: In Sec. 2, we discuss the infinite set of operators which violate diffeomorphism invariance and describe a set of terms which involve two derivatives of the metric which we will use as our test cases for this study. In Sec 3, we look at the linearized theory, find the propagator and calculate the bending of light. Because gauge non-invariant theories have extra degrees of freedom and could have ghosts we use the propagator analysis to probe for the existence of ghosts. We find that certain values of the parameters are required in order to be ghost-free. In Sec. 4, we start the matching to the PPN framework, which is carried out in detail in Sec. 5-8. In Sec. 9 we describe the resulting phenomenological constraint. Sec 10 is a brief summary. Several appendices describe auxiliary features of our treatment.

\section{FORMULATION}

Once one opens up the action to include non-covariant terms, the possibilities are myriad. When we organize the theory in an energy expansion, the action is ordered by powers of derivatives. In a theory with a metric, the metric $g_{\mu \nu}(x)$ is the primary field. There are no Lorentz invariant combinations of the metric without any derivatives, aside from the cosmological constant. In this case, the leading possible non-covariant terms in the action start with two derivatives. Terms with four derivatives or more would be suppressed at low energy. In this paper we study the two derivative modifications to the action. 
One should recognize that there might be other ways to test diffeomorphism breaking. For example, one can also include terms which violate Lorentz invariance [] $]$, as such breaking is also a form of diffeomorphism violation. Indeed there might be good reasons for including such terms for an emergent theory [7]. The breaking of Lorentz invariance has been already been studied in gravity [8] and we will study covariance breaking terms which are Lorentz invariant.

Moreover, if one gives up covariance and gives a special weight to a flat metric, it is possible that one could consider $h_{\mu \nu}(x)=g_{\mu \nu}(x)-\eta_{\mu \nu}$ as the primary field. In this case, there could be terms with zero derivatives in the actions with the leading effect being the Pauli-Fierz mass term [9]. This term has been heavily studied and is phenomenologically ruled-out at essentially any magnitude. When the mass is bigger than a critical fraction of the curvature, the van Dam-Veltman -Zakharov (vDVZ) discontinuity [10, 11] says that the predictions drastically disagree with general relativity. When the mass is smaller than the critical curvature the mass generates an intrinsic instability [12] in the spacetime in flat, de Sitter or Freidman-Robertson-Walker cosmology,. Only antide Sitter spaces escape these serious problems. However, anti-de Sitter space does not appear to be selected in Nature. So it appears that this form of diffeomorphism breaking must be identically zero. Combinations with higher powers of $h_{\mu \nu}(x)$ and zero derivatives should also be studied. Some of these are listed in Appendix A. However, we will turn our attention to the next order in the derivative expansion.

In the derivative expansion the next terms that would occur would be those with two derivatives of the metric. These are the ones that we study below. In addition there could be others with four derivatives of the metric. In principle these would be suppressed at low energy since the derivatives turn into factors of the graviton energy. Since we have no prior knowledge of the mass scale appearing in the energy expansion, these should be studied as well, but we reserve this for future work. We provide a classification of the symmetry breaking operators in the linear approximation up to sixth order in Appendix A.

Given these possibilities, we do not attempt a fully general analysis, but will look at some possibilities which have not been studied before and for which we can obtain a particularly tight bound. More general possibilities will be considered in future work.

In this section, we introduce a general second derivative Lagrangian involving the connection in ways that break the diffeomorphism invariance. This is a purely dynamical metric theory of gravity which assumes that [13] that there exists a symmetric metric. In addition, we also assume that all non-gravitational fields couple universally to the gravitational field.

Our general action takes the form

$$
S=\int d^{4} x \sqrt{-g} \mathcal{L}
$$

where

$$
\mathcal{L}=\frac{1}{16 \pi G}\left[R+\sum_{i=1}^{7} a_{i} \mathcal{L}_{i}\right]+\mathcal{L}_{m} .
$$

The first term above is the usual Einstein-Hilbert term, $\mathcal{L}_{m}$ is the matter Lagrangian, while $\mathcal{L}_{i}$ are the diffeomorphism-violating pieces

$$
\begin{aligned}
& \mathcal{L}_{1}=-g^{\mu \nu} \Gamma_{\mu \lambda}^{\alpha} \Gamma_{\nu \alpha}^{\lambda}, \quad \mathcal{L}_{2}=-g^{\mu \nu} \Gamma_{\mu \nu}^{\alpha} \Gamma_{\lambda \alpha}^{\lambda} \\
& \mathcal{L}_{3}=-g^{\alpha \gamma} g^{\beta \rho} g_{\mu \nu} \Gamma_{\alpha \beta}^{\mu} \Gamma_{\gamma \rho}^{\nu}, \quad \mathcal{L}_{4}=-g^{\alpha \gamma} g_{\beta \lambda} g^{\mu \nu} \Gamma_{\mu \nu}^{\lambda} \Gamma_{\gamma \alpha}^{\beta} \\
& \mathcal{L}_{5}=-g^{\alpha \beta} \Gamma_{\lambda \alpha}^{\lambda} \Gamma_{\mu \beta}^{\mu}, \quad \mathcal{L}_{6}=-g^{\mu \nu} \partial_{\nu} \Gamma_{\mu \lambda}^{\lambda} \\
& \mathcal{L}_{7}=-g^{\mu \nu} \partial_{\lambda} \Gamma_{\mu \nu}^{\lambda},
\end{aligned}
$$

and $\left\{a_{i}\right\}$ are small coefficients. The Greek indices run over four spacetime coordinates, and we take the Lorentz signature to be mostly positive. Notice that $\mathcal{L}_{E H}$ can be written as $-\mathcal{L}_{1}+\mathcal{L}_{2}+\mathcal{L}_{6}-\mathcal{L}_{7}$. Using integration by parts and the identities

$$
\partial_{\nu} g^{\rho \gamma}=-g^{\rho \alpha} \Gamma_{\nu \alpha}^{\gamma}-g^{\beta \gamma} \Gamma_{\beta \nu}^{\rho}, \partial_{\mu} \sqrt{-g}=\sqrt{-g} \Gamma_{\mu \nu}^{\nu}
$$

we obtain

$$
\mathcal{L}_{6}=\mathcal{L}_{2}+\text { surface term }, \quad \mathcal{L}_{7}=2 \mathcal{L}_{1}-\mathcal{L}_{2}+\text { surface term } .(5)
$$

Hence, $\mathcal{L}_{6}$ and $\mathcal{L}_{7}$ are not independent and we drop them in our analysis. Moreover, using the same identities, one can show that any other term of square derivative, e.g. $\sqrt{-g} \partial_{\gamma} g^{\alpha \beta} \partial^{\gamma} g_{\alpha \beta}$ or $\sqrt{-g} \partial_{\alpha} \partial_{\beta} g^{\alpha \beta}$, can be uniquely expressed as a linear combination of $\mathcal{L}_{1}$ through $\mathcal{L}_{5}$ modulo surface terms. This set is not unique beyond linear order, as once one has given up covariance one can have an infinite set of Lagrangians of the form $(\sqrt{-g})^{n} \mathcal{L}$ by adding extra powers of $(\sqrt{-g})^{n}$ starting at the same two derivative order. However, such modifications do not contribute new terms to the linear equations of motion.

The use of the connection in Eq. 3 is important for our matching to the PPN formalism. As we will see in the next section, this basis is larger than needed for the linear analysis keeping only the leading term in the expansion of the metric. However, the PPN formalism is sensitive to the higher order terms in the expansion of the metric. By using the connection in our operator basis, these higher order terms are part of expansion of the connection.

\section{LINEARIZED VERSION OF THE EQUATIONS OF MOTION}

Writing $g_{\mu \nu}=\eta_{\mu \nu}+h_{\mu \nu}$, and retaining only the quadratic contribution of $h$, we obtain

$$
\begin{array}{ll}
\mathcal{L}_{1}^{(2)}=-\frac{1}{4}\left(-T_{1}+2 T_{2}\right), & \mathcal{L}_{2}^{(2)}=-\frac{1}{4}\left(2 T_{3}-T_{4}\right) \\
\mathcal{L}_{3}^{(2)}=-\frac{1}{4}\left(3 T_{1}-2 T_{2}\right), & \mathcal{L}_{4}^{(2)}=-\frac{1}{4}\left(4 T_{2}-4 T_{3}+T_{4}\right) \\
\mathcal{L}_{5}^{(2)}=-\frac{T_{4}}{4}, &
\end{array}
$$


where

$$
\begin{aligned}
& T_{1}=\partial_{\gamma} h_{\alpha \beta} \partial^{\gamma} h^{\alpha \beta}, \quad T_{2}=\partial_{\gamma} h_{\alpha \beta} \partial^{\beta} h^{\alpha \gamma} \\
& T_{3}=\partial_{\alpha} h \partial_{\beta} h^{\alpha \beta}, \quad T_{4}=\partial_{\alpha} h \partial^{\alpha} h .
\end{aligned}
$$

Inspection of eq. (6) reveals that the quadratic Lagrangians $\mathcal{L}_{1}^{(2)}$ to $\mathcal{L}_{4}^{(2)}$ are independent. Hence, we conclude that the Lagrangians $\mathcal{L}_{1}$ to $\mathcal{L}_{4}$ are also independent.

Variation of the quadratic version of the Lagrangian (11) results in the linearized equations of motion

$$
\begin{aligned}
& \left(-1-a_{1}+3 a_{3}\right) \square h^{\alpha \beta}+\left(1+a_{1}-a_{3}+2 a_{4}\right)\left(\partial^{\alpha} \partial_{\gamma} h^{\beta \gamma}\right. \\
& \left.+\partial^{\beta} \partial_{\gamma} h^{\alpha \gamma}\right)+\left(-1+a_{2}-2 a_{4}\right) \eta^{\alpha \beta} \partial_{\mu} \partial_{\nu} h^{\mu \nu} \\
& +\left(-1+a_{2}-2 a_{4}\right) \partial^{\alpha} \partial^{\beta} h+\left(1-a_{2}+a_{4}+a_{5}\right) \eta^{\alpha \beta} \square h \\
& =16 \pi G T^{\alpha \beta},
\end{aligned}
$$

where $h=h_{\alpha}^{\alpha}$, and $T^{\alpha \beta}$ is the energy momentum tensor that results by varying the matter action with respect to $g_{\alpha \beta}$.

Throughout this work we assume that the energy momentum tensor of the matter fields is conserved. This translates in the linear theory into $\partial^{\alpha} T_{\alpha \beta}=0$. Taking the derivative of eq. (7) we obtain

$$
\begin{aligned}
& 2\left(a_{3}+a_{4}\right) \square \partial_{\beta} h^{\alpha \beta}+\left(a_{1}+a_{2}-a_{3}\right) \partial^{\alpha} \partial_{\mu} \partial_{\nu} h^{\mu \nu} \\
& +\left(a_{5}-a_{4}\right) \partial^{\alpha} \square h=0 .
\end{aligned}
$$

This equation is satisfied provided that we have either

- (i) Trivial case: all the coefficients $a_{1}$ to $a_{5}$ are set equal to zero, or they satisfy $a_{4}=a_{5}, a_{3}+a_{4}=0$ and $a_{1}+a_{2}-a_{3}=0$ ( the last two conditions result in $a_{1}+a_{2}-3 a_{3}-2 a_{4}=0$, see the comments below eq. (18)). This is the case of a diffeomorphism-invariant theory (in this case general relativity GR).

- (ii) General case: all coefficients $a_{1}$ to $a_{5}$ are different from zero. In this case the realization of eq. (8) can be guaranteed if we impose the constraints

$$
\partial_{\alpha} h^{\alpha \beta}=0, \quad \text { and } \quad a_{4}=a_{5} .
$$

The physical consequences to linear order (light bending) of this case is worked out below.

- (iii) Special case I: all coefficients are set to zero except $a_{4} \neq 0$. In this case we make use of the field redefinition $h^{\alpha \beta}=\bar{h}^{\alpha \beta}-\eta^{\alpha \beta} \bar{h} / 2$ to bring eq. (8) to the form

$$
\begin{aligned}
& -\square \bar{h}^{\alpha \beta}+\left(1+2 a_{4}\right)\left(\partial^{\alpha} \partial_{\gamma} \bar{h}^{\beta \gamma}+\partial^{\beta} \partial_{\gamma} \bar{h}^{\alpha \gamma}\right) \\
& -\left(1+2 a_{4}\right) \eta^{\alpha \beta} \partial_{\mu} \partial_{\nu} \bar{h}^{\mu \nu}=16 \pi G T^{\alpha \beta} .
\end{aligned}
$$

Then, imposing the condition $\partial_{\alpha} T^{\alpha \beta}=0$ we get the constraint $\partial_{\alpha} \bar{h}^{\alpha \beta}=0$. Using this constraint in eq. (10) we obtain $\square \bar{h}^{\alpha \beta}=16 \pi G T^{\alpha \beta}$. Hence, to linear order, setting $a_{4} \neq 0$ does not lead to any physical consequences beyond GR.
- (iv) Special case II: all the coefficients are set to zero except $a_{5} \neq 0$. In this case one can use the transformation $h^{\alpha \beta}=\bar{h}^{\alpha \beta}-\eta^{\alpha \beta} \bar{h} / 4$ to eliminate $a_{5}$. Again, this leads to no physical consequences on the linear level beyond GR.

It is important to notice here what has been stated in the literature that if we want a more general equation than Einstein's equation that respects Lorentz symmetry and reduces in the weak field limit to a second order equation, then we have to include other elements that are unrelated to the metric tensor or its derivatives, and we must give up the possibility of deriving Newton's theory as a limiting case (see e.g. [14]). The above statement is true only if we do not give up the diffeomorphism invariance as a fundamental symmetry of the underlying manifold. However, we have shown that breaking this symmetry can still result in a second order equation that has Newton's theory as a limiting case (see the discussion below) provided that we take the covariance breaking coefficients to be small enough.

Also, one may argue that we should run into troubles once we break the diffeomorphism invariance. The classical example is Pauli-Fierz massive gravity [9]. The argument is that since diffeomorphism symmetry is a dynamical symmetry, breaking it will excite the scalar modes that become strongly coupled even when we send the graviton mass to zero. This is the famous van DamVeltman-Zakharov (vDVZ) discontinuity [10, 11]. As we show below, this kind of discontinuity does not happen in our case. A simple explanation can be given here (see e.g. the first reference in [12]). The equation of motion for Pauli-Fierz massive gravity reads

$$
\begin{aligned}
& -\square h^{\alpha \beta}+\left(\partial^{\alpha} \partial_{\gamma} h^{\beta \gamma}+\partial^{\beta} \partial_{\gamma} h^{\alpha \gamma}\right)-\eta^{\alpha \beta} \partial_{\mu} \partial_{\nu} h^{\mu \nu} \\
& -\partial^{\alpha} \partial^{\beta} h+\eta^{\alpha \beta} \square h+m^{2}\left(h^{\alpha \beta}-\eta^{\alpha \beta} h\right) \\
& =16 \pi G T^{\alpha \beta} .
\end{aligned}
$$

Taking the divergence and trace of the above equation results in the five constraints

$$
\partial_{\alpha} h^{\alpha \beta}=\partial^{\beta} h, h=\frac{16 \pi G}{3 m^{2}} T .
$$

Along with the obvious behavior of $1 / \mathrm{m}^{2}$ in the limit of zero mass, the first constraint in (12) does not reduce to a guage condition consistent with equations of motion in the limit $m=0$. This behavior, which signals the presence of a problem, is absent in our case since the constraint $\partial_{\alpha} h^{\alpha \beta}=0$ reduces to a gauge condition as we send $a_{1}, a_{2}$, etc. to zero.

\subsection{The propagator and bending of light}

To further study the linearized theory, it is instructive to write down the graviton propagator. This can be accomplished by writing the quadratic Lagrangian in the 
form $h_{\mu \nu} O^{\mu \nu, \alpha \beta} h_{\alpha \beta}$, and finding the inverse of the operator $O$. To impose the constraint $\partial^{\alpha} h_{\alpha \beta}=0$, we insert in the Lagrangian a term $\Lambda\left(\partial^{\alpha} h_{\alpha \beta}\right)^{2}$, and take the limit $\Lambda \rightarrow \infty$ at the end of calculations. Hence, we find

$$
\begin{aligned}
D_{\mu \nu, \rho \sigma}(k)= & -A \eta_{\mu \nu} \eta_{\rho \sigma} / k^{2}+B\left(\eta_{\mu \rho} \eta_{\nu \sigma}+\eta_{\mu \sigma} \eta_{\nu \rho}\right) / k^{2} \\
& +A\left(\eta_{\mu \nu} k_{\rho} k_{\sigma}+\eta_{\rho \sigma} k_{\mu} k_{\nu}\right) / k^{4} \\
& -B\left(\eta_{\mu \rho} k_{\nu} k_{\sigma}+\eta_{\mu \sigma} k_{\nu} k_{\rho}+\eta_{\nu \sigma} k_{\mu} k_{\rho}\right. \\
& \left.+\eta_{\nu \rho} k_{\mu} k_{\sigma}\right) / k^{4}+C k_{\mu} k_{\nu} k_{\rho} k_{\sigma} / k^{6}
\end{aligned}
$$

where the constants $A, B, C$, and $D$ are given by

$$
\begin{aligned}
A & =\frac{1-a_{2}+2 a_{4}}{\left(1+a_{1}-3 a_{3}\right)\left(2-a_{1}-3 a_{2}+3 a_{3}+6 a_{4}\right)} \\
B & =\frac{1}{2\left(1+a_{1}-3 a_{3}\right)} \\
C & =\frac{1-a_{1}-2 a_{2}+3 a_{3}+4 a_{4}}{\left(1+a_{1}-3 a_{3}\right)\left(2-a_{1}-3 a_{2}+3 a_{3}+6 a_{4}\right)}
\end{aligned}
$$

and we have put $a_{4}=a_{5}$. As expected, the propagator is continuous to the GR result as we set $a_{1}=a_{2}=\ldots a_{4}=0$.

Now consider two particles with conserved energy momentum tensor $T_{(1)}^{\mu \nu}$ and $T_{(2)}^{\rho \sigma}$ interacting via the exchange of a graviton. The scattering amplitude is given by

$$
\begin{aligned}
& G T_{(1)}^{\mu \nu}(k) D_{\mu \nu, \rho \sigma}(k) T_{(2)}^{\rho \sigma}(k) \\
& =\frac{G}{k^{2}}\left(-A T_{(1)} T_{(2)}+2 B T_{(1)}^{\mu \nu} T_{(2) \mu \nu}\right) .
\end{aligned}
$$

The scattering amplitude between to chunks of nonrelativistic matter is proportional to

$$
\frac{G_{\text {eff }}}{2 k^{2}} T_{(1)}^{00} T_{(2)}^{00}
$$

where $G_{\text {eff }}=2 G(2 B-A)$. Now, taking $T_{(1)}^{\mu \nu}$ and $T_{(2)}^{\mu \nu}$ to be respectively the energy momentum tensor of the sun and photon we obtain the scattering amplitude, and hence the light bending effect

$$
\frac{G_{\mathrm{eff}}}{2 k^{2}} \frac{2-a_{1}-3 a_{2}+3 a_{3}+6 a_{4}}{1-a_{1}-2 a_{2}+3 a_{3}+4 a_{4}} .
$$

In appendix D, we obtain the same results using the PPN formalism.

\subsection{Ghost Analysis}

Finally, we consider the issue of a possible ghost instability in diffeomorphism-violating theories of gravity. Generally, ghosts can appear in effective field theories. However, they usually show up only at energies above the cut-off scale of the theory. A thorough analysis of the linearized version of the action (11) to search for such instabilities was given in [15] in the context of transversediffeomorphism theories of gravity [16]. Still, one can learn about these instabilities by studying the behavior of the momentum space propagator. A ghost propagator will have the opposite sign of a healthy degree of freedom. Hence, existence of an instability can be read from (13) as terms with the wrong sign. A quick way of doing this is by sandwiching the propagator between the energy momentum tensors of two sources as we did above, and then projecting out the transverse traceless part of the spin-2 particle. Hence, the scattering amplitude can be written as

$$
\begin{aligned}
& \frac{2 G B}{k^{2}}\left[T_{(1)}^{\mu \nu} T_{(2) \mu \nu}-\frac{1}{2} T_{(1)} T_{(2)}\right] \\
& -\frac{G B}{k^{2}} \frac{a_{1}+a_{2}-3 a_{3}-2 a_{4}}{2-a_{1}-3 a_{2}+3 a_{3}+6 a_{4}} T_{(1)} T_{(2)} .
\end{aligned}
$$

The first term is the usual spin- 2 graviton coupled to matter, while the second term represents massless interaction between conserved sources. This massless degree of freedom has a healthy kinetic term (not a ghost) provided that $a_{1}+a_{2}-3 a_{3}-2 a_{4} \leq 0$. The saturation of this inequality decouples the massless mode.

On the other hand, the analysis drawn in [15] showed that the Minkowskian vacuum in the linear version of (1) admits a linear classical instability for the vector modes unless we impose the constraint $a_{3}+a_{4}=0$. These modes couple to the derivative of the energy momentum tensor, and hence they do not show up in (18) since we are considering conserved sources. The constraint $a_{3}+a_{4}=0$ along with the saturation of the above inequality $\left(a_{1}+\right.$ $\left.a_{2}-3 a_{3}-2 a_{4}=0\right)$ restores the diffeomorphism invariance of the theory (GR)

We show below that experimental tests of gravity bound the values of $\left\{a_{i}\right\}$ to be of order $10^{-20}$. This suppresses any significance for ghosts or other classical instability, if any, at low energies. At higher energies, one expects higher order terms, possibly higher derivative Lagrangians, to contribute new degrees of freedom rendering the UV theory ghost free.

\section{NONLINEAR EQUATIONS OF MOTION}

The linear approximation is not sufficient for the PPN analysis that we are about to undertake. The iteration that is involved in that formalism mixes different powers of the linear field $h_{\mu \nu}$. Because our original Lagrangian was written in terms of the connection, we are able to include higher order terms using the original operators. Moreover at this stage we are going to restrict our treatment to one of the operators in our basis, $\mathcal{L}_{3}$. While we plan to report on it in future work, the treatment of the general case is very cumbersome and the use of this operator is sufficient to identify the strongest test, coming from the preferred frame parameter $\alpha_{3}$, and obtain a very strong constraint.

The nonlinear equations of motion of the system can be found using the Euler-Lagrange equations

$$
\frac{\partial \sqrt{-g} \mathcal{L}}{\partial g_{\alpha \beta}}-\partial_{\mu}\left(\frac{\partial \sqrt{-g} \mathcal{L}}{\partial g_{\alpha \beta, u}}\right)=0
$$


where the comma denotes ordinary derivative.

Taking into account the identities $g_{\alpha \beta} g^{\beta \gamma}=\delta_{\alpha}^{\gamma}$, and $\partial \sqrt{-g} / \partial g_{\alpha \beta}=\sqrt{-g} g^{\alpha \beta} / 2$ we obtain (from here on, we drop the subscript in $a_{3}$ to reduce notational clutter)

$$
R_{\mu \nu}-\frac{1}{2} g_{\mu \nu} R+a \mathcal{M}_{\mu \nu}=8 \pi G T_{\mu \nu},
$$

where $M_{\mu \nu}=\mathcal{B}_{\mu \nu}+\mathcal{D}_{\mu \nu}$, and the functions $\mathcal{B}_{\mu \nu}$ and $\mathcal{D}_{\mu \nu}$ are given by

$$
\begin{gathered}
\mathcal{B}_{\mu \nu}=-\frac{1}{2} g_{\mu \nu} g_{\alpha \beta} g^{\gamma \delta} g^{\epsilon \eta} \Gamma_{\gamma \epsilon}^{\alpha} \Gamma_{\delta \eta}^{\beta}+g^{\alpha \beta} g^{\gamma \delta} g_{\nu \phi} g_{\mu \epsilon} \Gamma_{\alpha \gamma}^{\epsilon} \Gamma_{\beta \delta}^{\phi} \\
+2 g^{\phi \epsilon} g^{\alpha \gamma} g_{\delta \epsilon} g_{\phi \beta} \Gamma_{\mu \alpha}^{\beta} \Gamma_{\nu \gamma}^{\delta} \\
\mathcal{D}_{\mu \nu}=\Gamma_{\alpha \lambda}^{\lambda} \mathcal{A}_{\mu \nu}^{\alpha}+\mathcal{A}_{\mu \nu, \alpha}^{\alpha},
\end{gathered}
$$

and

$$
\mathcal{A}_{\mu \nu}^{\alpha}=g^{\alpha \beta} g_{\gamma \mu} \Gamma_{\nu \beta}^{\gamma}+g^{\alpha \beta} g_{\gamma \nu} \Gamma_{\mu \beta}^{\gamma}-\Gamma_{\mu \nu}^{\alpha} .
$$

Since the equation of motion (201) is not invariant under general coordinate transformations, the existence of a solution requires that we impose a consistency condition, as we did in the linearized case. As usual, we assume that the energy momentum tensor is conserved, i.e. it satisfies $\nabla^{\mu} T_{\mu \nu}=0$, where $\nabla$ denotes the covariant derivative. In addition, we have from the geometry $\nabla^{\mu} G_{\mu \nu}=0$. Hence we must also have

$$
\nabla^{\mu} \mathcal{M}_{\mu \nu}=0
$$

which is the consistency condition.

In the rest of the paper, we use the parametrized post Newtonian (PPN) formalism to bound the numerical value of $a$ by comparing the outcomes of our theory of gravity with the experimental data.

\section{THE PPN FORMALISM}

To compare the different theories of gravity to experiments, we take the slow motion and weak field approximation. Such treatment is perturbative in nature and known as the post Newtonian formalism [17]. In this method, one expands in a small expansion parameter which is taken to be the velocity $v$ of a fluid element

$$
U \sim v^{2} \sim p / \rho \sim \Pi \sim \mathcal{O}(2),
$$

where $U$ is the Newtonian potential, $p$ is the pressure of the fluid, $\rho$ is its rest mass density, and $\Pi$ is the specific energy density (ratio of energy density to rest-mass density). The power of velocity $v$ is $\mathcal{O}(1), U$ is $\mathcal{O}(2)$, $\Pi$ is $\mathcal{O}(2)$ and $p$ is $\mathcal{O}(4)$. In addition, since the time evolution of a system is governed by the motion of its constituents, one has $\partial / \partial t \sim \vec{v} \cdot \nabla$, and hence

$$
\frac{|\partial / \partial t|}{|\partial / \partial x|} \sim \mathcal{O}(1) \text {. }
$$

To obtain the Newtonian limit, we need to solve for $g_{00}$ to $\mathcal{O}(2)$. Assuming $g_{00} \rightarrow 0$ far from the system we find $g_{00}=2 G_{\text {eff }} U$. Since we work in units in which the measured gravitational constant is unity, we set

$$
G_{\text {eff }} \equiv 1
$$

The post Newtonian corrections to the propagation of light may be found by solving for $g_{i j}$ to $\mathcal{O}(2)$. To this order, one can use the linearized equations of motion (7). However, for more involved experiments like the perihelion shift of Mercury, we need to know $g_{00}$ to $\mathcal{O}(4)$. To this order we work out the full PPN parameters using only $\mathcal{L}_{3}$ as an example.

In the following, we assume that the matter content is idealized as a perfect fluid, and hence the components of the energy momentum tensor to the relevant order are

$$
\begin{aligned}
T^{00} & =\rho\left(1+\Pi+v^{2}+2 U\right) \\
T^{0 i} & =\rho v^{i} \\
T^{i j} & =\rho v^{i} v^{j}+p \delta_{i j} .
\end{aligned}
$$

In addition, the metric will be constructed out of few gravitational potentials $U, U_{i j}, V_{i}, W_{i}, \Phi_{W}, \Phi_{1}, \Phi_{2}, \Phi_{3}$, $\Phi_{4}, \Phi_{5}, \mathcal{A}$, and $\mathcal{B}$. The reader can refer to appendix $\mathrm{B}$ for the explicit form of these potentials as well as the important differential relations they satisfy.

In the next section we review the setup used to solve systematically for $g_{\mu \nu}$ up to $\mathcal{O}(4)$.

\section{SETUP}

Far from the system under investigation, we expect that the metric tensor reduces to that of Minkowski space. Therefore, we expand the metric $g_{\mu \nu}$ about the Minkowskian background $\eta_{\mu \nu}=\{-1,1,1,1\}$ in powers of $v^{2}$

$$
\begin{aligned}
& g_{00}=-1+\stackrel{(2)}{g_{00}}+\stackrel{(4)}{g_{00}}+\ldots, \quad g_{i j}=\delta_{i j}+\stackrel{(2)}{g_{i j}}+\stackrel{(2)}{g_{i j}}+\ldots \\
& g_{0 i}=\stackrel{(3)}{g_{0 i}}+\stackrel{(5)}{g_{0 i}}+\ldots,
\end{aligned}
$$

where the Latin indices run over the spatial dimensions, and $g_{\mu \nu}^{(N)}$ is of order $v^{N}$. If we define the inverse metric as

$$
\begin{aligned}
g^{00} & =-1+\stackrel{(2)}{g^{00}}+\stackrel{(4)}{g^{00}}+\ldots, \quad g^{i j}=\delta_{i j}+\stackrel{(2)}{g^{i j}}+\stackrel{(4)}{g^{i j}}+\ldots \\
g^{0 i} & =\stackrel{(3)}{g^{0 i}}+\stackrel{(5)}{g^{0 i}}+\ldots,
\end{aligned}
$$

then using the identity $g_{\alpha \beta} g^{\beta \gamma}=\delta_{\alpha}^{\gamma}$ we find

$$
\stackrel{(2)}{g^{00}}=-\stackrel{(2)}{g_{00}}, \quad \stackrel{(2)}{g^{i j}}=-\stackrel{(2)}{g_{i j}}, \quad \stackrel{(3)}{g^{0 i}}=\stackrel{(3)}{g_{0 i}} .
$$


The components of the affine connections are given in appendix $\mathrm{C}$ by (C1). Also, the components of $R_{\mu \nu}$ and $\mathcal{M}_{\mu \nu}$ have the form

$$
\begin{aligned}
& R_{00}=\stackrel{(2)}{R}_{00}+\stackrel{(4)}{R}_{00}+\ldots, \quad \mathcal{M}_{00}=\stackrel{(2)}{\mathcal{M}}_{00}+\stackrel{(4)}{\mathcal{M}}_{00}+\ldots \\
& R_{0 i}=\stackrel{(3)}{R_{0 i}}+\stackrel{(5)}{R_{0 i}}+\ldots, \quad \mathcal{M}_{0 i}=\stackrel{(3)}{\mathcal{M}}_{0 i}+\stackrel{(5)}{(5)}_{0 i}+\ldots \\
& R_{i j}=\stackrel{(2)}{R_{00}}+\stackrel{(4)}{R}_{i j}+\ldots, \quad \mathcal{M}_{i j}=\stackrel{(2)}{\mathcal{M}}_{i j}+\stackrel{(4)}{\mathcal{M}}_{i j}+\ldots .
\end{aligned}
$$

At this stage, it is more convenient to take the trace of (20) and rewrite it in the form

$$
R_{\mu \nu}+a\left(\mathcal{M}_{\mu \nu}-\frac{1}{2} g_{\mu \nu} \mathcal{M}\right)=8 \pi G\left(T_{\mu \nu}-\frac{1}{2} g_{\mu \nu} T\right)
$$

Plugging the expansion (32) into eq. (33) we obtain, to the relevant order,

$$
\begin{aligned}
& \stackrel{(2)}{R_{00}}+\frac{a}{2}\left(\stackrel{(2)}{\mathcal{M}}_{00}+\stackrel{(2)}{\mathcal{M}}_{i i}\right)=4 \pi G T^{00} \\
& \stackrel{(2)}{R_{i j}}+a\left(\stackrel{(2)}{\mathcal{M}}_{i j}+\frac{1}{2} \delta_{i j} \stackrel{(2)}{\mathcal{M}}_{00}-\frac{1}{2} \delta_{i j} \stackrel{(2)}{\mathcal{M}}_{k k}\right)=4 \pi G \delta_{i j} \stackrel{(2)}{T}^{00} \\
& \stackrel{(3)}{R_{0 i}}+a \stackrel{(3)}{\mathcal{M}_{0 i}}=-8 \pi G \stackrel{(3)}{T^{0 i}} \\
& \stackrel{(4)}{R} 00+\frac{a}{2}\left(\stackrel{(4)}{\mathcal{M}}_{00}+\stackrel{(4)}{\mathcal{M}}_{i i}-\stackrel{(2)}{g}_{00} \stackrel{(2)}{(2)}_{i i}-\stackrel{(2)}{g}_{i j} \stackrel{(2)}{\mathcal{M}}_{i j}\right) \\
& =4 \pi G\left(\stackrel{(4)}{T^{00}}+\stackrel{(4)}{i i}-2 \stackrel{(2)}{g_{00}} T^{00}\right),
\end{aligned}
$$

where the summation is indicated in $\mathcal{M}_{i i}$.

Finally, eqs. (34) have to be supplemented with the constraint (24) to every order, i.e.

$$
\begin{aligned}
& \nabla^{i} \stackrel{(2)}{\mathcal{M}}_{i j}=0 \\
& \nabla^{0} \stackrel{(2)}{\mathcal{M}}_{00}+\nabla^{i} \stackrel{(3)}{\mathcal{M}}_{0 i}=0 \\
& \nabla^{0} \stackrel{(3)}{\mathcal{M}}_{0 i}+\nabla^{i} \stackrel{(4)}{\mathcal{M}}_{i j}=0 .
\end{aligned}
$$

\section{LOWER ORDER SOLUTIONS}

In this section, we investigate the Newtonian, $g_{00}^{(2)}$, and the first post Newtonian, $g_{i j}^{(2)}$ and $g_{0 i}^{(3)}$, limits for our theory of gravity. By direct calculations, we obtain the lower order expressions for $R$ and $\mathcal{M}$ as given by (C2) in appendix C. In addition, the first two constraints in (35) results in

$$
\stackrel{(2)}{g_{i j, j}}=0, \quad \stackrel{(3)}{g_{i 0, i}}-\stackrel{(2)}{g_{00,0}}=0,
$$

which greatly simplifies the subsequent analysis.
Plugging eq. (C2) into eq. (33) and using (36) we find

$$
\begin{aligned}
& (1-3 a) \nabla^{2} \stackrel{(2)}{g_{00}}=-16 \pi G \frac{1+3 a}{2+3 a} \stackrel{(2)}{T_{00}} \\
& -g_{00, i j}^{(2)}+g_{k k, i j}^{(2)}+(1-3 a) g_{00, k k}^{(2)}=16 \pi G \frac{\delta_{i j}}{2+3 a} \stackrel{(2)}{T} \\
& -g_{00,0 i}^{(2)}+g_{k k, 0 i}^{(2)}+(1-3 a) g_{0 i, k k}^{(3)}=-16 \pi G \stackrel{(3)}{T}_{0 i}
\end{aligned}
$$

The solution of $g_{00}^{(2)}$ is given by

$$
\stackrel{(2)}{g_{00}}=\frac{2 G(1+3 a)}{(1-3 a)(1+3 a / 2)} U .
$$

Because the PPN formalism works in units in which the gravitational constant is unity, we must set

$$
\frac{G(1+3 a)}{(1-3 a)(1+3 a / 2)}=1 \text {. }
$$

Hence, the normalized solutions of $g_{00}^{(2)}, g_{i j}^{(2)}$, and $g_{0 i}^{(2)}$ takes the form

$$
\begin{aligned}
\stackrel{(2)}{g_{00}} & =2 U \\
\stackrel{(2)}{g_{i j}} & =\frac{1}{1+3 a}\left(U \delta_{i j}+U_{i j}\right) \\
\stackrel{(3)}{g_{0 i}} & =-\frac{1}{1+3 a}\left[(3+6 a) V_{i}+W_{i}\right] .
\end{aligned}
$$

\section{HIGHER ORDER SOLUTIONS}

Finding $g_{00}$ to $\mathcal{O}(4)$ is a cumbersome step since it involves all the lower order solutions. Moreover, the requirement that the solution satisfies the constraint (24) makes it a long and tedious procedure.

The solution of $g_{00}^{(4)}$ can be obtained from the last eq. in (34). This involves the higher order perturbations of $R_{00}$, $\mathcal{M}_{00}$ and $\mathcal{M}_{i i}$. The expressions for these functions are given in eq. (C3). Although we do not need the explicit value of $g_{i j}^{(4)}$ in the PPN formalism, the appearance of this term in $\mathcal{M}_{i i}^{(4)}$ necessitates, in general, a simultaneous solution for $g_{i j}^{(4)}$ and $g_{00}^{(4)} \cdot{ }^{1}$ In turn, this adds more to the complexity of the problem by forcing us to feed the system in (34) with the $i-j$ equation to $\mathcal{O}(4)$. However, since the parameter $a$ is small, we will be interested only in solutions to first order in $a$. To this end, one can solve for the terms $g_{i j}^{(4)}$ to the zeroth order of $a$ using only the general relativity (GR) part, i.e. using $R_{i j}^{(4)}$ and neglecting completely the contribution from $\mathcal{M}_{i j}^{(4)}$. At

\footnotetext{
${ }^{1}$ In fact, it is clear from eq. C3) that we only need the combination $\left(3 g_{i i, k k}^{(4)}-2 \stackrel{(4)}{g_{i k, i k}}\right)$.
} 
the end, we substitute the result in (34) when trying to find $g_{00}^{(4)}$. ${ }^{2}$ This introduces an error of $\mathcal{O}\left(a^{2}\right)$ in our calculations. The solution of $g_{i j}^{(4)}$ in GR was first given by Chandrasekhar and Nutku [18] in the PPN gauge. However, to be consistent we should get an answer that obeys the constraint (36). We work out the details of these calculations in appendix E. Using the results of appendix $\mathrm{E}$ along with the dictionary of appendix $\mathrm{F}$ we find

$$
\begin{aligned}
\stackrel{(4)}{R}_{00}= & \nabla^{2}\left[-\frac{1}{2} \stackrel{(4)}{g_{00}}-\frac{1}{2} U^{2}+\frac{7 / 2+3 a}{1+3 a} \Phi_{2}+\frac{\Phi_{W}}{2(1+3 a)}\right. \\
& \left.-\frac{3 a}{1+3 a}\left(\mathcal{A}+\mathcal{B}-\Phi_{1}\right)\right] \\
\stackrel{(M}{M}_{00}= & \nabla^{2}\left[\frac{1}{2} \stackrel{(4)}{g_{00}}-\frac{35}{16} U^{2}-\frac{11}{8} \Phi_{W}-\frac{3}{2} \Phi_{1}-\frac{23}{8} \Phi_{2}\right. \\
& \left.+\frac{3}{2} \mathcal{A}+\frac{3}{2} \mathcal{B}+\frac{1}{16} U_{i j} U_{i j}\right] \\
\mathcal{M}_{i i}^{(4)}= & \nabla^{2}\left[\frac{115}{16} U^{2}-\frac{1}{8} \Phi_{W}+6 \Phi_{1}-\frac{69}{8} \Phi_{2}+8 \Phi_{3}\right. \\
& \left.+2 \mathcal{A}+2 \mathcal{B}+\frac{7}{16} U_{i j} U_{i j}+\mathcal{V}\right],
\end{aligned}
$$

where $\mathcal{V}=E_{i, i}$, and $E_{i}$ are arbitrary functions associated with $g_{i j}^{(4)}$ as explained in appendix E. Moreover, the r.h.s of the last equation of (34) reads

$$
\begin{aligned}
& 4 \pi G\left(\stackrel{(4)}{T^{00}}+\stackrel{(4)}{T^{i i}}-2 \stackrel{(2)}{g_{00}} \stackrel{(2)}{00}\right)=\left(1-\frac{9}{2} a\right) \times \\
& \times \nabla^{2}\left[-2 \Phi_{1}+2 \Phi_{2}-\Phi_{3}-3 \Phi_{4}\right] .
\end{aligned}
$$

Now using the last eq. in (34), and solving for $g_{00}^{(4)}$ to the first order in $a$ we obtain

$$
\begin{aligned}
{\stackrel{(4)}{g_{00}}=}^{(} & \left(-1+\frac{15}{2} a\right) U^{2}+\left(1-\frac{5}{2} a\right) \Phi_{W} \\
& +\left(4-\frac{11}{2} a\right) \Phi_{1}+\left(3-\frac{59}{2} a\right) \Phi_{2} \\
& +2 \Phi_{3}+(6-24 a) \Phi_{4}-\frac{5}{2} a(\mathcal{A}+\mathcal{B}) \\
& +\frac{a}{2} U_{i j} U_{i j}+a \mathcal{V} .
\end{aligned}
$$

Next, we impose the constraint (24), and derive the equation that determines the function $\mathcal{V}$.

\subsection{Determining $\mathcal{V}$}

To find the condition that determines $\mathcal{V}$, we use the constraint (24) to $\mathcal{O}(4)$, as in the last eq. of (35). Taking the derivative of the aforementioned equation with

\footnotetext{
${ }^{2}$ We would like to thank Clifford M. Will for bringing this point to our attention.
}

respect to $x^{i}$, and using eqs. (E1), (E4), and (E5) we obtain

$$
\begin{aligned}
& \nabla^{4} \mathcal{V}+\frac{1}{4} \nabla^{2} S_{i i}-\stackrel{\mathcal{M}}{0 i, 0 i}_{(3)}-\left(\stackrel{(2)}{\Gamma}_{j j}^{k} \stackrel{(2)}{(2)}_{k i}+\stackrel{(2)}{k}_{k j}^{(2)} \mathcal{M}_{i j}\right. \\
& \left.+\stackrel{(2)}{g}_{j k} \stackrel{(2)}{M}_{i j, k}-\stackrel{(2)}{\Gamma_{00}^{k}} \stackrel{(2)}{\mathcal{M}}_{k i}-\stackrel{(2)}{\Gamma_{0 i}^{0}} \stackrel{(2)}{\mathcal{M}_{00}}\right)_{, i} \\
& +\mathcal{B}_{i j, i j}^{(4)}+\mathcal{P}_{i j, i j}^{(4)}=0,
\end{aligned}
$$

where $S_{i i}$ is given by (E7), and the rest of the quantities are given in appendix C. Using the dictionary in (F2), and solving for $\mathcal{V}$ we find after long, yet straightforward calculations

$$
\begin{aligned}
\mathcal{V}= & -\frac{83}{16} U^{2}-\frac{5}{4} \Phi_{W}-\frac{45}{8} \Phi_{1}+\frac{7}{2} \Phi_{2}-2 \Phi_{3}+\frac{33}{4} \Phi_{4} \\
& +\frac{5}{4} \Phi_{5}-\frac{1}{2} \mathcal{A}+\frac{29}{8} \mathcal{B}-\frac{1}{16} U_{i j} U_{i j} .
\end{aligned}
$$

In the next section we read off the PPN parameters and constraint the value of $a$.

\section{PPN PARAMETER VALUES AND INTERPRETATION}

To extract the PPN parameters one has to bring the the form of the metric to the standard PPN metric by means of a gauge transformation. However, since we are dealing with a theory that breaks the diffeomorphism invariance, one does not expect that such transformation will always respect the constraint (24). We can overcome this problem, simply, by transforming the standard PPN metric to the gauge that satisfies (24). This transformation is given by

$$
\begin{aligned}
g_{i j}^{\mathrm{PPN}} & =g_{i j}-2 \lambda_{2} \chi_{, i j} \\
g_{0 i}^{\mathrm{PPN}} & =g_{0 i}-\left(\lambda_{1}+\lambda_{2}\right)\left(V_{i}-W_{i}\right) \\
g_{00}^{\mathrm{PPN}} & =g_{00}-2 \lambda_{2}\left(U^{2}+\Phi_{W}-\Phi_{2}\right)-2 \lambda_{1}\left(\mathcal{A}+\mathcal{B}-\Phi_{1}\right),
\end{aligned}
$$

where the expressions for $g_{\mu \nu}^{\mathrm{PPN}}$ are functions of ten PPN parameters as given in [17]. Comparing eqs. (46) and (43) we can read off the values of the gauge parameters $\lambda_{1}$ and $\lambda_{2}$, as well as the PPN parameters as given in table 【

The parameters $\gamma$ and $\beta$ are Eddington-RobertsonSchiff parameters used to describe the classical tests of theories of gravity; namely the deflection of light, time delay and perihelion shift. The parameter $\xi$ is non-zero in any theory of gravity that predicts preferred-location effects. Also, $\alpha_{1}, \alpha_{2}$ and $\alpha_{3}$ measure whether or not the theory predicts post-Newtonian preferred-frame effects.

\footnotetext{
${ }^{3}$ The reader can refer to [19] for a recent and extended review of
} 


\begin{tabular}{|c|c|c|c|}
\hline parameter & value & effect & limit \\
\hline \hline$\gamma-1$ & $-3 \mathrm{a}$ & time delay & $2.3 \times 10^{-5}$ \\
\hline & & light deflection & $4 \times 10^{-4}$ \\
\hline$\beta-1$ & $-\frac{85}{32} a$ & perihelion shift & $3 \times 10^{-3}$ \\
\hline & & Nordtvedt effect & $2.3 \times 10^{-4}$ \\
\hline$\xi$ & $\frac{3}{8} a$ & earth tides & $10^{-3}$ \\
\hline$\alpha_{1}$ & 0 & orbital polarization & $10^{-4}$ \\
\hline$\alpha_{2}$ & 0 & orbital polarization & $4 \times 10^{-7}$ \\
\hline$\alpha_{3}$ & $-\frac{65}{8} a$ & orbital polarization & $4 \times 10^{-20}$ \\
\hline$\zeta_{1}$ & $\frac{39}{8} a$ & - & $2 \times 10^{-2}$ \\
\hline$\zeta_{2}$ & $-\frac{179}{16} a$ & binary acceleration & $4 \times 10^{-5}$ \\
\hline$\zeta_{3}$ & $-a$ & Newtons 3rd law & $10 \times 10^{-8}$ \\
\hline$\zeta_{4}$ & $\frac{5}{8} a$ & - & - \\
\hline
\end{tabular}

TABLE I: The values and limits on the PPN parameters [19].

When one attempts to devise integral conservation laws, we search for a quantity $\Theta^{\mu \nu}$ which reduces to $T^{\mu \nu}$ in flat spacetime and satisfies $\partial_{\mu} \Theta^{\mu \nu}=0$. It was shown in [20] that such quantity can exist only if all the parameters $\left\{\alpha_{3}, \zeta_{1}, \zeta_{2}, \zeta_{3}, \zeta_{4}\right\}$ are identically zero. Non-zero values of these parameters measure the extent at which a theory of gravity predicts violations of conservation of total energy and momentum. Notice that the parameter $\alpha_{3}$ plays a dual role, both as a conservation law and preferred-frame parameter.

A bound on $\alpha_{3}$, of $4 \times 10^{-20}$ was reported in 21, 22] from the period derivatives of 21 millisecond pulsars. This small bound puts severe constraint on the value of $\left\{a_{i}\right\}$, and in turn on the diffeomorphism-violating Lagrangians.

Finally, it is worth noting that our result for $g_{00}$ to $\mathcal{O}(4)$ contains additional potentials $U_{i j} U_{i j}$ and $\Phi_{5}$ that are not present in the standard PPN formalism. It would be interesting to devise experiments aimed to measure the effects of such terms in gravitational systems.

\section{SUMMARY}

Our work helps to quantify the physical content of general covariance. We have allowed for the possibility of small violations of diffeomorphism invariance through a class of operators with two derivatives of the metric. An analysis to linear order produced modest constraints from light bending. However, we have used the PPN formalism to bound the non-invariance of a sample operator in this basis, which produces a far stronger constraint. By far the strongest result comes from the absence of preferred frame effects in pulsars, and leads to the constraint that the dimensionless parameter $a$ must be less than $10^{-20}$ of gravitational strength.

Tests of diffeomorphism invariance are of interest in its own right. We want to have quantitative probes of

the different tests of GR. this fundamental property. Moreover, we have argued that this constraint is relevant for theories in which general relativity is an emergent phenomenon from a more fundamental theory that lacks a fundamental version of diffeomorphism invariance. There are many directions that extensions of this initial investigation can go and we feel that the topic deserves further study.

\section{Acknowledgements}

This work has been supported in part by the NSF grants PHY- 055304 and PHY - 0855119, and in part by the Foundational Questions Institute. We would like to thank T.J. Blackburn, Luca Grisa and Lorenzo Sorbo for interesting discussions. Also, we would like to thank Clifford Will for useful communications.

\section{APPENDIX A: OPERATORS OF THE LINEARIZED THEORY}

In this appendix we write down the lower, marginal and higher dimensional operators for a linearized theory of gravity where we expand $g_{\mu \nu}=\eta_{\mu \nu}+h_{\mu \nu}$.

- Dimension-2 operators

The lowest dimension operators can only contain powers of the field, without derivatives. At leading order,

$$
h^{2}, h^{\mu \nu} h_{\mu \nu}
$$

The Pauli-Fierz mass term is

$$
\frac{m^{2}}{4}\left(h^{\mu \nu} h_{\mu \nu}-h^{2}\right)
$$

- Dimension-3 operators

$$
h^{3}, h h_{\beta}^{\alpha} h_{\alpha}^{\beta}, h_{\alpha}^{\mu} h_{\nu}^{\alpha} h_{\mu}^{\nu}
$$

- Dimension-4 operators

At dimension- 4 we start to have the possibility of operators with two derivatives, starting the next series in the derivative expansion. Those without derivatives are

$$
h^{4}, h^{2} h_{\beta}^{\alpha} h_{\alpha}^{\beta}, h h_{\beta}^{\alpha} h_{\gamma}^{\beta} h_{\alpha}^{\gamma}, h_{\beta}^{\alpha} h_{\gamma}^{\beta} h_{\delta}^{\gamma} h_{\alpha}^{\delta}
$$

while those with two derivatives are the set

$$
\mathcal{C}^{(4)}=\left\{\partial_{\mu} h^{\alpha \beta} \partial^{\mu} h_{\alpha \beta}, \partial_{\alpha} h^{\alpha \beta} \partial_{\gamma} h_{\beta}^{\gamma}, \partial_{\alpha} h \partial^{\alpha} h, \partial_{\alpha} h \partial^{\beta} h_{\beta}^{\alpha}\right\}
$$


- Dimension-5 operators

At this order we stop listing the series with zero derivatives and show the next order terms in the series with two derivatives. These all have three powers of the field and occur in the combinations

$$
\mathcal{C}^{4} h, \mathcal{C}^{(4) \alpha \beta} h_{\alpha \beta}
$$

where $\mathcal{C}^{4}$ is defined above and

$$
\begin{aligned}
& \mathcal{C}^{(4) \alpha \beta}=\left\{\quad \partial^{\alpha} h^{\mu \nu} \partial^{\beta} h_{\mu \nu}, \partial^{\alpha} h \partial^{\beta} h, \partial_{\mu} h^{\alpha \beta} \partial^{\mu} h,\right. \\
& \partial^{\nu} h^{\alpha \beta} \partial^{\mu} h_{\mu \nu}, \partial^{\nu} h_{\mu}^{\alpha} \partial_{\nu} h^{\mu \beta}, \partial_{\nu} h^{\alpha \nu} \partial_{\mu} h^{\mu \beta}, \\
& \partial^{\alpha} h^{\mu \nu} \partial_{\nu} h_{\mu}^{\beta}, \partial^{\alpha} h \partial^{\mu} h_{\mu}^{\beta}, \partial^{\mu} h \partial^{\alpha} h_{\mu}^{\beta}, \\
& \left.\partial_{\nu} h^{\mu \nu} \partial^{\alpha} h_{\mu}^{\beta}\right\}
\end{aligned}
$$

- Dimension-6 operators

At sixth order, the four derivative series starts. We here list only those with four derivatives and two powers of the field.

$$
\begin{aligned}
& \partial_{\alpha} \partial_{\beta} h \partial^{\alpha} \partial^{\beta} h, \partial_{\alpha} \partial_{\beta} h_{\mu \nu} \partial^{\alpha} \partial^{\beta} h^{\mu \nu}, \partial_{\alpha} \partial_{\beta} h^{\mu \nu} \partial_{\mu} \partial_{\nu} h^{\alpha \beta}, \\
& \partial_{\alpha} \partial_{\beta} h_{\mu \nu} \partial^{\alpha} \partial^{\nu} h_{\beta}^{\mu} .
\end{aligned}
$$

\section{APPENDIX B: GRAVITATIONAL POTENTIALS}

In this appendix, we list the various gravitational potentials used to construct the metric.

$$
\begin{aligned}
U & =\int d^{3} x^{\prime} \frac{\rho^{\prime}}{\left|\vec{x}-\vec{x}^{\prime}\right|} \\
U_{i j} & =\int d^{3} x^{\prime} \frac{\rho^{\prime}\left(x-x^{\prime}\right)_{i}\left(x-x^{\prime}\right)_{j}}{\left|\vec{x}-\vec{x}^{\prime}\right|^{3}} \\
V_{i} & =\int d^{3} x^{\prime} \frac{\rho^{\prime} v_{i}^{\prime}}{\left|\vec{x}-\vec{x}^{\prime}\right|} \\
W_{i} & =\int d^{3} x^{\prime} \frac{\rho^{\prime} \vec{v}^{\prime} \cdot\left(\vec{x}-\vec{x}^{\prime}\right)\left(x-x^{\prime}\right)_{i}}{\left|\vec{x}-\vec{x}^{\prime}\right|^{3}} \\
\Phi_{1} & =\int d^{3} x^{\prime} \frac{\rho^{\prime} v^{\prime 2}}{\left|\vec{x}-\vec{x}^{\prime}\right|}, \Phi_{2}=\int d^{3} x^{\prime} \frac{\rho^{\prime} U^{\prime}}{\left|\vec{x}-\vec{x}^{\prime}\right|} \\
\Phi_{3} & =\int d^{3} x^{\prime} \frac{\rho^{\prime} \Pi^{\prime}}{\left|\vec{x}-\vec{x}^{\prime}\right|}, \Phi_{4}=\int d^{3} x^{\prime} \frac{p^{\prime}}{\left|\vec{x}-\vec{x}^{\prime}\right|} \\
\mathcal{A} & =\int d^{3} x^{\prime} \frac{\rho^{\prime}\left[\vec{v}^{\prime} \cdot\left(\vec{x}-\vec{x}^{\prime}\right)\right]^{2}}{\left|\vec{x}-\vec{x}^{\prime}\right|^{3}} \\
\mathcal{B} & =\int d^{3} x^{\prime} \frac{\rho^{\prime} d \vec{v}^{\prime} / d t \cdot\left(\vec{x}-\vec{x}^{\prime}\right)}{\left|\vec{x}-\vec{x}^{\prime}\right|} \\
\Phi_{W} & =\int d^{3} x^{\prime} \rho^{\prime} \rho^{\prime \prime} \frac{\vec{x}-\vec{x}^{\prime}}{\left|\vec{x}-\vec{x}^{\prime}\right|^{3}} \cdot\left(\frac{\vec{x}^{\prime}-\vec{x}^{\prime \prime}}{\left|\vec{x}-\vec{x}^{\prime \prime}\right|}-\frac{\vec{x}-\vec{x}^{\prime \prime}}{\left|\vec{x}^{\prime}-\vec{x}^{\prime \prime}\right|}\right) .
\end{aligned}
$$

These potentials satisfy the differential relations

$$
\begin{aligned}
& \nabla^{2} V_{i}=-4 \pi \rho v_{i}, \quad V_{i, i}=-U_{, 0} \\
& \nabla^{2} \Phi_{1}=-4 \pi \rho v^{2}, \quad \nabla^{2} \Phi_{2}=-4 \pi \rho U \\
& \nabla^{2} \Phi_{3}=-4 \pi \rho \Pi, \quad \nabla^{2} \Phi_{4}=-4 \pi p \\
& \nabla^{2}\left(\Phi_{W}+2 U^{2}-3 \Phi_{2}\right)=2 \chi_{, i j} U_{, i j} \\
& \chi_{, 00}=\mathcal{A}+\mathcal{B}-\Phi_{1},
\end{aligned}
$$

where

$$
\begin{aligned}
\chi & =-\int d^{3} x^{\prime} \rho^{\prime}\left|\vec{x}-\vec{x}^{\prime}\right| \\
\chi_{, i j} & =-\delta_{i j} U+U_{i j}, \quad \nabla^{2} \chi=-2 U .
\end{aligned}
$$

In addition, consider the potential

$$
\psi_{i}=\int d^{3} x^{\prime} \frac{U_{i j}^{\prime} \rho_{, j}^{\prime}}{\left|\vec{x}-\vec{x}^{\prime}\right|},
$$

such that $\nabla^{2} \psi_{i}=-4 \pi U_{i j} \rho_{, j}$. Hence, we define the potential $\Phi_{5}$ as

$$
\nabla^{2} \psi_{i, i} \equiv \nabla^{4} \Phi_{5}=4 \pi \rho_{, i} U_{, i}-4 \pi U_{i j} \rho_{, i j} .
$$

\section{APPENDIX C: EXPRESSIONS USED THROUGHOUT THE PAPER}

In this appendix we give the form of the different expressions used throughout this paper.

The components of the affine connections are

$$
\begin{aligned}
& \stackrel{(2)}{\Gamma_{00}^{i}}=-\frac{1}{2} \stackrel{(2)}{g}{ }_{00, i}, \quad \stackrel{(4)}{\Gamma_{00}^{i}}=-\frac{1}{2} \stackrel{(4)}{g}_{00, i}^{(3)}+\stackrel{(3)}{g_{0 i, 0}}+\frac{1}{2} \stackrel{(2)}{g}{ }_{i j} g_{00, j}^{(2)} \\
& \stackrel{(3)}{\Gamma_{0 j}^{i}}=\frac{1}{2}\left[g_{i 0, j}^{(3)}+\stackrel{(2)}{g_{i j, 0}}-g_{j 0, i}^{(3)}\right], \quad \stackrel{(3)}{\Gamma_{00}^{0}}=-\frac{1}{2} g_{00,0}^{(2)} \\
& \stackrel{(2)}{\Gamma_{j k}^{i}}=\frac{1}{2}\left[\stackrel{(2)}{g_{i j, k}}+\stackrel{(2)}{g_{i k, j}}-\stackrel{(2)}{g_{j k, i}}\right], \quad \stackrel{(2)}{\Gamma_{0 i}^{0}}=-\frac{1}{2} \stackrel{(2)}{g_{00, i}} \\
& \stackrel{(4)}{\Gamma_{j k}^{i}}=\frac{1}{2} \delta^{i p}\left[\stackrel{(4)}{g_{p j, k}}+\stackrel{(4)}{g_{p k, i}}-g_{i k, p}^{(4)}\right] \\
& -\frac{1}{2} \stackrel{(2)}{g}\left[g_{i p}^{(2)}\left[g_{p i, k}^{(2)}+g_{p k, i}^{(2)}-g_{i k, p}\right]\right. \text {. }
\end{aligned}
$$

The lower order expressions for $R$ and $\mathcal{M}$ read

$$
\begin{aligned}
\stackrel{(2)}{R_{00}} & =-\frac{1}{2} \stackrel{(2)}{g_{00, i i}}, \quad \stackrel{(2)}{\mathcal{M}_{00}}=\frac{3}{2} g_{00, i i}^{(2)} \\
\stackrel{(2)}{R_{i j}} & =\frac{1}{2}\left[g_{00, i j}^{(2)}-g_{k k, i j}^{(2)}+g_{i k, k j}^{(2)}+g_{k j, k i}^{(2)}-g_{i j, k k}^{(2)}\right] \\
\stackrel{(2)}{M}_{i j} & =\frac{1}{2}\left[-g_{i k, k j}^{(2)}-g_{j k, k i}^{(2)}+3 g_{i j, k k}^{(2)}\right] \\
\stackrel{(3)}{R_{i 0}} & =\frac{1}{2}\left[-g_{j j, 0 i}^{(2)}+g_{j 0, i j}^{(3)}+g_{i j, j 0}^{(2)}-g_{i 0, k k}^{(3)}\right] \\
\stackrel{(3)}{M}_{i 0} & =\frac{3}{2} g_{i 0, k k}^{(2)} .
\end{aligned}
$$


The higher order perturbations of $R$ and $\mathcal{M}$ are given by (the constraint (36) being imposed)

$$
\begin{aligned}
& \stackrel{(4)}{R_{00}}=-\frac{1}{2} \stackrel{(2)}{g i i, 00}^{(2)} g_{i 0, i 0}^{(3)}-\frac{1}{2} g_{00, k k}^{(4)}+\frac{1}{2} \stackrel{(2)}{g_{i j}} g_{00, i j}^{(2)} \\
& -\frac{1}{4} \stackrel{(2)}{g_{00, i}} \stackrel{(2)}{g_{00, i}}-\frac{1}{4} \stackrel{(2)}{g_{00, i}}{ }_{g_{j j, i}}^{(2)} \\
& \stackrel{(4)}{\mathcal{M}}_{00}=-\frac{15}{2}{\stackrel{(2)}{g_{00, i}}}_{g_{00, i}}^{(2)}+3 \stackrel{(2)}{g_{i i, j}} \stackrel{(2)}{(2)}_{00, j}-2 \stackrel{(2)}{g_{00,00}}+2 \stackrel{(4)}{g_{00, j j}} \\
& -4 g_{0 j, 0 j}^{(3)}-6 \stackrel{(2)}{g}{ }_{i j} g_{00, i j}^{(2)}+\frac{3}{2} \stackrel{(2)}{g_{i j, k}} \stackrel{(2)}{g_{i j, k}}-{\stackrel{(2)}{g}{ }_{j, i}{ }^{(2)} g_{i j, k}}^{(2)} \\
& \stackrel{(4)}{\mathcal{M}}_{i i}=-\frac{3}{4}{\stackrel{(2)}{g_{i i, j}}}_{g_{00, j}}^{(2)}+\frac{3}{4}{\stackrel{(2)}{g_{k k, j}}}_{g_{i i, j}}^{(2)}-\frac{3}{2} g_{i i, 00}^{(2)}+g_{0 i, 0 i}^{(3)} \\
& -\frac{3}{8} \stackrel{(2)}{(2)}_{00, i}^{(2)} g_{00, i}+\frac{9}{8} \stackrel{(2)}{(2)} g_{i k, j}^{(2)} g_{i k, j}-\frac{3}{4} g_{i j, k}^{(2)} g_{k j, i}^{(2)} \\
& -\frac{3}{2} \stackrel{(2)}{g_{k p}} g_{i i, k p}^{(2)}+\frac{1}{2}\left(3 \stackrel{(4)}{g_{i i, k k}}-2 \stackrel{(4)}{g_{i k, i k}}\right) \text {, }
\end{aligned}
$$

where the summation is implied in $\stackrel{(4)}{(4)}_{i \text {. }}$

The functions $\mathcal{B}_{i j}$ and $\mathcal{P}_{i j}$ defined in (44) are given by

$$
\begin{aligned}
& \stackrel{(4)}{\mathcal{B}}_{i j}=-\delta_{i j}\left(\frac{3}{8} g_{00, k}^{(2)} g_{00, k}^{(2)}+\frac{3}{8} g_{n k, m}^{(2)} g_{n k, m}^{(2)}\right. \\
& \left.-\frac{1}{4} g_{n m, k}^{(2)} \stackrel{(2)}{g_{k m, n}}\right)+\stackrel{(2)}{\Gamma_{00}^{i}} \stackrel{(2)}{\Gamma_{00}^{j}}+\stackrel{(2)}{\Gamma_{k m}^{i}} \stackrel{(2)}{\Gamma_{k m}^{j}} \\
& +2 \stackrel{(2)}{\Gamma_{i k}^{m}} \stackrel{(2)}{\Gamma}_{j k}^{m}+2 \stackrel{(2)}{\Gamma_{i 0}^{0}} \stackrel{(2)}{\Gamma}_{j 0}^{0} \\
& \stackrel{(2)}{\mathcal{P}_{i j}}=\stackrel{(2)}{\Gamma^{0}} \stackrel{(2)}{A}_{i j}^{k}+\stackrel{(2)}{\Gamma_{k m}^{m}} \stackrel{(2)}{A}_{i j}^{k}+\stackrel{(3)}{A_{i j, 0}^{0}}+\stackrel{(4)}{\mathcal{Q}_{i j, k}^{k}}
\end{aligned}
$$

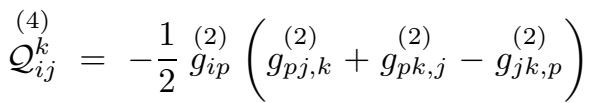

$$
\begin{aligned}
& -\frac{1}{2} \stackrel{(2)}{g}\left(\stackrel{(2)}{g_{p i, k}}+g_{p k, i}^{(2)}-g_{i k, p}^{(2)}\right) \\
& +\frac{1}{2} \stackrel{(2)}{g}_{k p}\left(g_{p i, j}^{(2)}+g_{p j, i}^{(2)}-g_{i j, p}^{(2)}\right) \\
& +\left(\stackrel{(2)}{g}_{n i} \stackrel{(2)}{\Gamma}_{j k}^{n}+\stackrel{(2)}{g_{n j}} \stackrel{(2)}{\Gamma_{i k}^{n}}-\stackrel{(2)}{g_{k m}} \stackrel{(2)}{\Gamma_{j m}^{i}}-\stackrel{(2)}{g_{k m}} \stackrel{(2)}{\Gamma_{i m}^{j}}\right) .
\end{aligned}
$$

\section{APPENDIX D: LINEARIZED VERSION OF THE EQUATIONS OF MOTION}

One can use the linearized version of the equations of motion (7) along with the constraint $\partial_{\beta} h^{\alpha \beta}=0$ to solve for $g_{i j}$ to $\mathcal{O}(2)$ and $g_{0 i}$ to $\mathcal{O}(3)$. ${ }^{4}$ To this end, we take

\footnotetext{
${ }^{4}$ Notice that $h_{00}=g_{00}^{(2)}, h_{i j}=g_{i j}^{(2)}$, and $h_{0 i}=g_{0 i}^{(3)}$.
}

the trace of (7), and write $h$ in terms of $T$ to find

$$
\begin{aligned}
& \left(1+a_{1}-3 a_{3}\right) \square h^{\alpha \beta}+\left(1-a_{2}+2 a_{4}\right) \partial^{\alpha} \partial^{\beta} h \\
& =-16 \pi G\left(\stackrel{(2)}{ }^{\alpha \beta}-A \eta^{\alpha \beta} \stackrel{(2)}{T}\right)
\end{aligned}
$$

where

$$
A=\frac{1-a_{2}+a_{4}+a_{5}}{2-a_{1}-3 a_{2}+3 a_{3}+2 a_{4}+4 a_{5}} .
$$

Solving for the $h_{00}$ component we get

$$
h_{00}=2 \alpha U,
$$

where

$$
\alpha=\frac{2 G\left(1-a_{1}-2 a_{2}+3 a_{3}+a_{4}+3 a_{5}\right)}{\left(1+a_{1}-3 a_{3}\right)\left(2-a_{1}-3 a_{2}+3 a_{3}+2 a_{4}+4 a_{5}\right)} .
$$

The most general solution of $h_{i j}$ is given by

$$
h_{i j}=\sigma_{1} \delta_{i j} U+\sigma_{2} U_{i j}
$$

where $\sigma_{1}$ and $\sigma_{2}$ are constants to be determined. Using the constraint $\partial_{i} h_{i j}$ we find $\sigma_{1}=\sigma_{2}$. Then, substituting into eq. (D1) we find that the following two equations

$$
2 \sigma_{1}\left(1+a_{1}-3 a_{3}\right)=\frac{4 G\left(1-a_{2}+a_{4}+a_{5}\right)}{2-a_{1}-3 a_{2}+3 a_{3}+2 a_{4}+4 a_{5}},
$$

and

$$
\sigma_{1}\left(-1+a_{1}-3 a_{3}+2 a_{2}-4 a_{4}\right)=-\alpha\left(1-a_{2}+2 a_{4}\right),
$$

have to be satisfied simultaneously. This can be true only if we take $a_{4}=a_{5}$. This is exactly what we found before in eq. (9). Setting $\alpha=1$, we obtain the normalized value of $\sigma_{1}$

$$
\sigma_{1}=\frac{1-a_{2}+2 a_{4}}{1-a_{1}-2 a_{2}+3 a_{3}+4 a_{4}}
$$

from which we immediately read the PPN parameter $\gamma$

$$
\gamma=\frac{1-a_{2}+2 a_{4}}{1-a_{1}-2 a_{2}+3 a_{3}+4 a_{4}},
$$

which reduces in the limiting case $a_{1}=a_{2} \ldots=a_{5}=0$ to the GR result. The deflection of light is proportional to $\gamma+1$, which gives (17).

\section{APPENDIX E: SOLVING FOR $\left(3 \stackrel{(4)}{g_{i i, k k}}-2 \underset{g_{i k, i k}^{(4)}}{(4)}\right)$}

To find $g_{i j}$ to $\mathcal{O}(4)$, we need to write the $i-j$ component of the equation of motion (33) to $\mathcal{O}(4)$. However, since we are interested in $g_{00}^{(4)}$ to first order in $a$, we can solve for $g_{i j}^{(4)}$ to zeroth order in $a$. Hence, 
the $i-j$ component of (33) reduces to the GR result $R_{i j}+\mathcal{O}(a)=8 \pi G\left(T_{i j}-g_{i j} T / 2\right)$. Moreover, the solution of $g_{i j}^{(4)}$ should respect the constraint (36).

The $i-j$ component of the Ricci tensor can be evaluated to the fourth order to find

$$
g_{i j, k k}^{(4)}+\stackrel{(4)}{g_{k k, i j}}-\stackrel{(4)}{g_{i k, k j}}-g_{j k, k i}^{(4)}=S_{i j}
$$

where $S_{i j}$ is a complicated function of the various potentials. Contracting eq. (E1) results in

$$
\nabla^{2} \stackrel{(4)}{g}_{i i}-g_{i j, i j}^{(4)}=\frac{1}{2} S_{i i}
$$

while differentiating it with respect to $j$ gives

$$
\left(\nabla^{2} \stackrel{(4)}{g}_{i i}-g_{i j, i j}^{(4)}\right)_{, m}=S_{m k, k}
$$

From eqs. (E2) and (E3) we obtain the integrability condition

$$
\left(S_{i j}-\frac{1}{2} \delta_{i j} S_{k k}\right)_{, i}=0 .
$$

It was shown by Chandrasekhar and Nutku [18] that this condition is indeed satisfied in GR in the PPN gauge. Since GR is a diffeomorphism-invariant theory, we conclude that this condition still holds when using the constraint (36). It was also shown in [] that the solution of eq. (E2) is given by

$$
\nabla^{2} \stackrel{(4)}{g} i j=S_{i j}+E_{i, j}+E_{j, i}
$$

where $E_{i}$ are arbitrary functions. ${ }^{5}$ Now, using eqs. (E2) and (E5) we get

$$
3 g_{i i, k k}^{(4)}-2 \stackrel{(4)}{g_{i k, i k}}=2 S_{i i}+2 E_{i, i}
$$

where

$$
\begin{aligned}
S_{i i}= & \frac{1}{2} g_{00, i}^{(2)} g_{00, i}^{(2)}+\stackrel{(2)}{g}_{00} g_{00, i i}^{(2)}+\frac{3}{2} g_{k p, i}^{(2)} g_{k p, i}^{(2)}+\stackrel{(2)}{g}_{k p} g_{k p, i i}^{(2)} \\
& +2 \stackrel{(2)}{g_{i i, 00}}+\stackrel{(2)}{g_{k p}} g_{i i, p k}^{(2)}-g_{i k, m}^{(2)} g_{i m, k}^{(2)}-\frac{1}{2} g_{m m, k}^{(2)} g_{i i, k}^{(2)} \\
& +\frac{1}{2} g_{00, k}^{(2)} g_{i i, k}^{(2)}-2 \stackrel{(3)}{g_{0 i, 0 i}}+g_{00, k k}^{(4)} \\
& -8 \pi\left(-T^{i i}+3 T^{00}-3 \stackrel{(2)}{g}_{00}^{(2)} T^{00}+\stackrel{(2)}{g}_{i i}^{(2)} T^{00}\right) \cdot(\mathrm{E} 7)
\end{aligned}
$$

\footnotetext{
${ }^{5}$ Strictly speaking, since we are using only GR, the constraint (36) is not more than a gauge fixing to $\mathcal{O}(2)$, and $E_{i}$ accounts for the gauge freedom to $\mathcal{O}(4)$. Although we are free to fix a gauge and hence the values of $E_{i}$ in case we were dealing only with GR, the values of these functions will be determined upon using the consistency condition (24) to $\mathcal{O}(4)$.
}

The value of $\stackrel{(4)}{g}_{00}$ can be calculated using $R_{00}+\mathcal{O}(a)=$ $8 \pi G\left(T_{00}-g_{00} T / 2\right)$, and imposing the constraint (36) to find

$$
\stackrel{(4)}{g_{00}}=-U^{2}+\Phi_{W}+4 \Phi_{1}-4 \Phi_{2}+2 \Phi_{3}+6 \Phi_{4} .
$$

Hence, using the dictionary in appendix $\mathrm{F}$ we finally obtain

$$
\begin{aligned}
& S_{i i}=\nabla^{2}\left[\frac{25}{4} U^{2}+\frac{5}{2} \Phi_{W}+8 \Phi_{1}+\frac{15}{2} \Phi_{2}+8 \Phi_{3}\right. \\
& \left.+\frac{1}{4} U_{i j} U_{i j}\right] \text {. }
\end{aligned}
$$

Writing $E_{i}$ as the sum of the gradient and curl of a scalar and vector, i.e. $E_{i}=\mathcal{V}_{, i}+(\nabla \times \vec{A})_{, i}$, we find

$$
E_{i, i}=\nabla^{2} \mathcal{V}
$$

\section{APPENDIX F: DICTIONARY}

In this appendix, we give a dictionary for the different combinations that appear in our formalism.

Using the differential relations in appendix B, we obtain to the zeroth order of $a$

$$
\begin{aligned}
& { }_{g_{i j, k}}^{(2)}{ }_{g_{i j, k}}^{(2)}=\nabla^{2}\left[\frac{9}{2} U^{2}+\Phi_{W}-7 \Phi_{2}+\frac{1}{2} U_{i j} U_{i j}\right] \\
& \stackrel{(2)}{g_{i j}} \underset{i j, k k}{(2)}=\nabla^{2}\left[-2 U^{2}-\Phi_{W}+7 \Phi_{2}\right] \\
& \stackrel{(2)}{g_{j k}} g_{i i, j k}^{(2)}=\nabla^{2}\left[4 U^{2}+2 \Phi_{W}+2 \Phi_{2}\right] \\
& g_{0 i, 0 i}^{(3)}=\nabla^{2}\left[\Phi_{1}-\mathcal{A}-\mathcal{B}\right] \\
& \stackrel{(2)}{g_{00, i}} \stackrel{(2)}{g_{00, i}}=\nabla^{2}\left[2 U^{2}-4 \Phi_{2}\right] \\
& \stackrel{(2)}{g_{i i, k}} \stackrel{(2)}{g_{j j, k}}=\nabla^{2}\left[8 U^{2}-16 \Phi_{2}\right] \\
& g_{00,00}^{(2)}=\nabla^{2}\left[\Phi_{1}-\mathcal{A}-\mathcal{B}\right] \\
& g_{i k, j}^{(2)} g_{i j, k}^{(2)}=\nabla^{2}\left[\frac{1}{2} U^{2}+\Phi_{W}+\Phi_{2}+\frac{1}{2} U_{i j} U_{i j}\right] \\
& \stackrel{(2)}{g_{00, i}} \stackrel{(2)}{g_{k k, i}}=\nabla^{2}\left[4 U^{2}-8 \Phi_{2}\right] \\
& \stackrel{(2)}{g_{00}} g_{00, k k}^{(2)}=\nabla^{2}\left[4 \Phi_{2}\right] \\
& \stackrel{(2)}{g_{i j}} g_{00, i j}^{(2)}=\nabla^{2}\left[2 U^{2}+\Phi_{W}+\Phi_{2}\right] \text {. }
\end{aligned}
$$


One can also show

$$
\begin{aligned}
U_{, i j} U_{, i j}= & \nabla^{4}\left(\frac{1}{4} U^{2}-\frac{1}{2} \Phi_{2}\right)+4 \pi \rho_{, i} U_{, i} \\
\chi_{, i j k} U_{, i j k}= & \nabla^{4}\left(\frac{3}{4} U^{2}+\frac{1}{4} \Phi_{W}-\frac{5}{4} \Phi_{2}\right) \\
& +4 \pi \rho_{, i} U_{, i}+2 \pi U_{i j} \rho_{, i j}-2 \pi U \nabla^{2} \rho \\
\chi_{, i j k m} \chi_{, i j k m}= & \nabla^{4}\left(\frac{11}{4} U^{2}+\Phi_{W}-4 \Phi_{2}+\frac{1}{4} U_{i j} U_{i j}\right) \\
& +8 \pi \rho_{, i} U_{, i}+4 \pi U_{i j} \rho_{, i j}-4 \pi U \nabla^{2} \rho \\
\nabla^{4} \Phi_{2}= & -8 \pi \rho_{, i} U_{, i}+16 \pi^{2} \rho^{2}-4 \pi U \nabla^{2} \rho \\
\nabla^{4} \Phi_{4}= & \left(\frac{1}{2} \Phi_{1}-\frac{1}{2} \beta\right)-4 \pi \rho_{, i} U_{, i}+16 \pi^{2} \rho^{2} .
\end{aligned}
$$

[1] J. F. Donoghue, "General Relativity As An Effective Field Theory: The Leading Quantum Corrections," Phys. Rev. D 50, 3874 (1994) arXiv:gr-qc/9405057. J. F. Donoghue, "Introduction to the Effective Field Theory Description of Gravity," arXiv:gr-qc/9512024. Published in the procedings of the Advanced School On Effective Theories, Almunecar, Spain, ed by F. Cornet and M.J. Hererro, (World Scientific, 1997)

[2] M. Gaul and C. Rovelli, "Loop quantum gravity and the meaning of diffeomorphism invariance," Lect. Notes Phys. 541, 277 (2000) arXiv:gr-qc/9910079.

L. Smolin, "The case for background independence," arXiv:hep-th/0507235

[3] J. Ambjorn, R. Janik, W. Westra and S. Zohren, "The emergence of background geometry from quantum fluctuations," Phys. Lett. B 641, 94 (2006) arXiv:gr-qc/0607013.

J. Ambjorn, J. Jurkiewicz and R. Loll, "Quantum gravity, or the art of building spacetime," arXiv:hep-th/0604212.

J. Ambjorn, J. Jurkiewicz and R. Loll, "Emergence of a 4D world from causal quantum gravity," Phys. Rev. Lett. 93, 131301 (2004) arXiv:hep-th/0404156].

Z. C. Gu and X. G. Wen, "Emergence of helicity +/- 2 modes (gravitons) from qbit models," arXiv:0907.1203 [gr-qc].

M. Levin and X. G. Wen, "Colloquium: Photons and electrons as emergent phenomena," Rev. Mod. Phys. 77, 871 (2005).

M. Levin and X. G. Wen, "Quantum ether: Photons and electrons from a rotor model," Phys. Rev. B 73 (2006) 035122 arXiv:hep-th/0507118.

X. G. Wen, "Artificial light and quantum order in systems of screened dipoles," Phys. Rev. B 68, 115413 (2003) arXiv:cond-mat/0210040]. S. S. Lee, "Emergence of gravity from interacting simplices," Int. J. Mod. Phys. A 24, 4271 (2009) arXiv:gr-qc/0609107. C. Xu, "Algebraic liquid phase with soft graviton excitations," arXiv:cond-mat/0602443

N. Seiberg, "Emergent spacetime,"
arXiv:hep-th/0601234

L. Sindoni, F. Girelli and S. Liberati, "Emergent gravitational dynamics in Bose-Einstein condensates," arXiv:0909.5391 [gr-qc].

S. Liberati, F. Girelli and L. Sindoni, "Analogue Models for Emergent Gravity," arXiv:0909.3834 [gr-qc].

S. Weinfurtner, M. Visser, P. Jain and C. W. Gardiner, "On the phenomenon of emergent spacetimes: An instruction guide for experimental cosmology," PoS QG-PH, 044 (2007) arXiv:0804.1346 [gr-qc]].

G. E. Volovik, "Emergent physics: Fermi point scenario," Phil. Trans. Roy. Soc. Lond. A 366, 2935 (2008) arXiv:0801.0724 [gr-qc]].

F. R. Klinkhamer and G. E. Volovik, "Coexisting vacua and effective gravity," Phys. Lett. A 347, 8 (2005) arXiv:gr-qc/0503090.

G. E. Volovik, "Superfluid analogies of cosmological phenomena," Phys. Rept. 351, 195 (2001) arXiv:gr-qc/0005091.

T. Konopka, F. Markopoulou and S. Severini, "Quantum Graphity: a model of emergent locality," Phys. Rev. D 77, 104029 (2008) arXiv:0801.0861 [hep-th]].

O. Dreyer, "Emergent general relativity," arXiv:gr-qc/0604075

[4] P. Horava, "Quantum Gravity at a Lifshitz Point," Phys. Rev. D 79, 084008 (2009) arXiv:0901.3775 [hep-th]].

[5] S. Weinberg and E. Witten, "Limits On Massless Particles," Phys. Lett. B 96, 59 (1980).

[6] V. A. Kostelecky and J. Tasson, "Prospects for Large Relativity Violations in Matter-Gravity Couplings," Phys. Rev. Lett. 102, 010402 (2009) arXiv:0810.1459 [gr-qc]].

R. Bluhm, S. H. Fung and V. A. Kostelecky, "Spontaneous Lorentz and Diffeomorphism Violation, Massive Modes, and Gravity," Phys. Rev. D 77, 065020 (2008) arXiv:0712.4119 [hep-th]].

V. A. Kostelecky and R. Potting, "Gravity from spontaneous Lorentz violation," Phys. Rev. D 79, 065018 (2009) arXiv:0901.0662 [gr-qc]].

[7] M. Anber, U, Aydemir and J. F. Donoghue "Gauge non- 
invariance, Lorentz violation and the phenomenology of emergent gauge theories" (in preparation).

T. P. Sotiriou, M. Visser and S. Weinfurtner, "Phenomenologically viable Lorentz-violating quantum gravity," Phys. Rev. Lett. 102, 251601 (2009) arXiv:0904.4464 [hep-th]].

M. Visser, "Lorentz symmetry breaking as a quantum field theory regulator," Phys. Rev. D 80, 025011 (2009) arXiv:0902.0590 [hep-th]].

[8] Q. G. Bailey and V. A. Kostelecky, "Signals for Lorentz violation in post-Newtonian gravity," Phys. Rev. D 74, 045001 (2006) arXiv:gr-qc/0603030.

[9] M. Fierz and W. Pauli, "On relativistic wave equations for particles of arbitrary spin in an electromagnetic field," Proc. Roy. Soc. Lond. A 173, 211 (1939).

[10] H. van Dam and M. J. G. Veltman, "Massive And Massless Yang-Mills And Gravitational Fields," Nucl. Phys. B 22, 397 (1970).

[11] V. I. Zakharov, "Linearized gravitation theory and the graviton mass," JETP Lett. 12, 312 (1970) [Pisma Zh. Eksp. Teor. Fiz. 12, 447 (1970)].

[12] D. G. Boulware and S. Deser, "Can gravitation have a finite range?," Phys. Rev. D 6, 3368 (1972). A. Higuchi, "FORBIDDEN MASS RANGE FOR SPIN2 FIELD THEORY IN DE SITTER SPACE-TIME," Nucl. Phys. B 282, 397 (1987).

S. Deser and A. Waldron, "Stability of massive cosmological gravitons," Phys. Lett. B 508, 347 (2001) arXiv:hep-th/0103255.

L. Grisa and L. Sorbo, "Pauli-Fierz Gravitons on Friedmann-Robertson-Walker Background,"
arXiv:0905.3391 [hep-th].

[13] Kip S. Thorne, David L. Lee, and Alan P. Lightman, "Foundations for a Theory of Gravitation Theories," Phys. Rev. D 7, 3563 (1973)

[14] Steven Weinberg, "Gravitation and Cosmology," 1972, John Wiley and Sons.

[15] E. Alvarez, D. Blas, J. Garriga and E. Verdaguer, "Transverse Fierz-Pauli symmetry," Nucl. Phys. B 756, 148 (2006) arXiv:hep-th/0606019.

[16] E. Alvarez, "Can one tell Einstein's unimodular theory from Einstein's general relativity?," JHEP 0503, 002 (2005) arXiv:hep-th/0501146.

[17] C. M. Will, "Theory and experiment in gravitational physics," Cambridge, UK: Univ. Pr. (1993) 380 p

[18] S. Chandrasekhar and Yavuz Nutku, "The second postNewotnian equations of hydrodynamics in general relativity," The Astrophysical Journal, 158, 1969.

[19] "Clifford M. Will," "The Confrontation between General Relativity and Experiment," Living Rev. Relativity 9, (2006), 3. URL http://www.livingreviews.org/lrr-2006-3

[20] D. L. Lee, A. P. Lightman and W. T. Ni, "Conservation laws and variational principles in metric theories of gravity," Phys. Rev. D 10, 1685 (1974).

[21] J. F. Bell and T. Damour, "A new test of conservation laws and Lorentz invariance in relativistic gravity," Class. Quant. Grav. 13, 3121 (1996) arXiv:gr-qc/9606062.

[22] I. H. Stairs et al., "Discovery of Three Wide-orbit Binary Pulsars: Implications for Binary Evolution and Equivalence Principles," Astrophys. J. 632, 1060 (2005) arXiv:astro-ph/0506188. 